\title{
Gastrectomia Vertical e Cirurgia de Bypass Gástrico em Y de Roux: complicações cirúrgicas e metabólicas tardias
}

\author{
Vertical Gastrectomy and Roux-en-Y Gastric Bypass Surgery: Iate surgical and metabolic \\ complications
}

\author{
Gastrectomía vertical y cirugía de bypass gástrico en Y de Roux: complicaciones \\ metabólicas y quirúrgicas tardías
}

\begin{abstract}
Letícia Jeber Marra $^{1 *}$, Amanda Silva Vasconcelos ${ }^{2}$, Ana Carolina Cardozo Fernandes ${ }^{3}$, Catharine Menezes Mota ${ }^{4}$, Eduarda Vieira de Andrade Leitão ${ }^{3}$, Laura Souza de Jesus ${ }^{4}$, Mariana Lima Vale ${ }^{5}$, Nayara Lima de Miranda ${ }^{6}$, Sabrina Rodrigues Carvalho ${ }^{4}$, Henrique Moura Parreira ${ }^{3}$.
\end{abstract}

\section{RESUMO}

Objetivo: Descrever e discutir as complicações pós-operatórias tardias mais comuns em cirurgia bariátrica, a partir da análise de duas técnicas amplamente utilizadas atualmente que são a Gastrectomia Vertical e a Cirurgia de Bypass Gástrico em Y de Roux. Revisão bibliográfica: A cirurgia bariátrica é vista como o tratamento de maior eficácia para a obesidade grave, determinada por $I M C>40 \mathrm{~kg} / \mathrm{m}^{2}$ ou $I M C$ superior a $35 \mathrm{~kg} / \mathrm{m}^{2}$ correlacionada à comorbidades. Essa intervenção apresenta baixa mortalidade, porém as complicações são recorrentes. A fístula na linha de grampeamento, o sangramento gastrointestinal, a obstrução intestinal e a ulceração marginal são ressaltadas como os eventos tardios mais frequentes. Dentre as complicações metabólicas e nutricionais as mais evidenciadas são a Síndrome de Dumping, a doença do refluxo gastroesofágico, a colelitíase e a diabetes mellitus. Considerações finais: A cirurgia de Bypass Gástrico em Y de Roux é considerada padrão-ouro, apesar de não haver concordância sobre qual intervenção deve ser executada em cada caso. Para a escolha do procedimento, devem ser analisados os riscos e examinados fatores como grau de obesidade e contraindicações existentes.

Palavras-chave: Complicações pós-operatórias, Cirurgia bariátrica, Gastrectomia.

\section{ABSTRACT}

Objective: To describe and discuss the most common late postoperative complications in bariatric surgery, based on analysis of two techniques widely used today, which are Vertical Gastrectomy and Roux-en-Y Gastric Bypass Surgery. Bibliographic review: Bariatric surgery is seen as the most effective treatment for severe obesity, determined by a BMI $>40 \mathrm{~kg} / \mathrm{m}^{2}$ or a BMI higher than $35 \mathrm{~kg} / \mathrm{m}^{2}$ correlated with comorbidities. This intervention has low mortality, but complications are recurrent. Staple line fistula, gastrointestinal bleeding, intestinal obstruction and marginal ulceration are highlighted as the most frequent late events. Among the metabolic and nutritional complications the most evident are Dumping Syndrome, gastroesophageal reflux disease, cholelithiasis and diabetes mellitus. Final considerations: Roux-en-Y Gastric Bypass Surgery is considered the gold standard, although there is no agreement on which intervention should be performed in each case. To choose the procedure, the risks must be analyzed and factors such as degree of obesity and against existing indications must be examined.

Key words: Postoperative complications, Bariatric surgery, c.

\footnotetext{
${ }^{1}$ Faculdade Ciências Médicas de Minas Gerais (FCMMG), Belo Horizonte - MG.

*E-mail: leticiajeberm@gmail.com

${ }^{2}$ Centro Universitário FACISA (UNIFACISA), Campina Grande - PB.

${ }^{3}$ Centro universitário de Caratinga (UNEC), Caratinga - MG.

${ }^{4}$ Universidade Nilton Lins (UNL), Manaus - AM.

${ }^{5}$ Centro Universitário INTA (UNINTA), Sobral - CE.

${ }^{6}$ Centro Universitário Ingá (Uningá), Maringá - PR.
} 


\section{RESUMEN}

Objetivo: Describir y discutir las complicaciones postoperatorias tardías más comunes en la cirugía bariátrica, a partir del análisis de das técnicas actualmente muy utilizadas que son la Gastrectomía Vertical y el Bypass Gástrico en Y de Roux. Revisión de la literatura: La cirugía bariátrica se considera el tratamiento más eficaz para la obesidad severa determinada por un $\mathrm{IMC}>40 \mathrm{~kg} / \mathrm{m} 2$ o un $\mathrm{IMC}>35 \mathrm{~kg} / \mathrm{m} 2$ correlacionado con comorbilidades. Esta intervención tiene una baja mortalidad, pero las complicaciones son recurrentes. La fístula en la línea de grapado, la hemorragia gastrointestinal, la obstrucción intestinal y la ulceración marginal se destacan como los eventos tardíos más frecuentes. Entre las complicaciones metabólicas y nutricionales, el síndrome de dumping, la enfermedad por reflujo gastroesofágico, la colelitiasis y la diabetes mellitus son las más evidentes. Consideraciones finales: El bypass gástrico en $Y$ de Roux se considera el estándar de oro, aunque no hay acuerdo sobre qué intervención debe realizarse en cada caso. Para la elección del procedimiento, se deben analizar los riesgos y examinar factores como el grado de obesidad y las contraindicaciones existentes.

Palabras clave: Complicaciones postoperatorias, Cirugía bariátrica, Gastrectomía.

\section{INTRODUÇÃO}

A obesidade é uma doença crônica, causada pelo acúmulo excessivo de gordura no corpo que pode gerar danos à saúde. É caracterizada por um Índice de Massa Corporal (IMC) superior a $30 \mathrm{~kg} / \mathrm{m}^{2}$, tanto para homens, quanto para mulheres. Além disso, de acordo com a Organização Mundial da Saúde (OMS), no período entre 1975 e 2016, a prevalência mundial da obesidade praticamente triplicou. As projeções apontam que, se não tratada da forma correta, a obesidade em 2025 terá atingido $18 \%$ dos homens e $21 \%$ das mulheres em todo o mundo. Ademais, tal patologia é tida como um sério problema de saúde pública, configurando a principal causa de mortes preveníveis (OMS, 2020; ZHANG Q, et al., 2020).

Dentre outras razões, a obesidade é uma doença preocupante devido seu potencial de aumentar os riscos de desenvolvimento de comorbidades como a hipertensão arterial, osteoartrite, doença renal crônica, diabetes mellitus e outras doenças importantes. $O$ tratamento desta patologia envolve desde a abordagem nutricional, bem como o uso de medicamentos e a prática de exercícios físicos. No entanto, vários pacientes não respondem a esses tratamentos clínicos e o tratamento cirúrgico passa a ser uma opção (KOVESDY CP, et al., 2017; ZHANG Q, et al., 2020).

A cirurgia bariátrica é considerada o tratamento de maior eficácia para a obesidade grave, caracterizada por um $I M C>40 \mathrm{~kg} / \mathrm{m}^{2}$, e está indicada também para pacientes cujo IMC é superior a $35 \mathrm{~kg} / \mathrm{m}^{2} \mathrm{com}$ comorbidades associadas, como diabetes, apneia do sono, hipertensão arterial, entre outras. Os benefícios dessa intervenção envolvem, além da perda ponderal, a melhoria de desordens cardiovasculares e metabólicas. Atualmente, as técnicas cirúrgicas de Bypass Gástrico em Y de Roux (BGYR) e a Gastrectomia Vertical (GV) são as cirurgias bariátricas e metabólicas mais comumente executadas (VILALLONGA R, et al., 2020).

A cirurgia de Bypass Gástrico em Y de Roux é uma modalidade restritiva e disabsortiva e é considerada como padrão ouro para o tratamento da obesidade grave, com sua taxa de sucesso bem estabelecida. Nessa técnica, é realizado o grampeamento de parte do estômago e um desvio do intestino inicial, promovendo, além da restrição de volume, o aumento de hormônios que dão saciedade (SCHLOTTMANN $F$; BUXHOEVEDEN R, 2018; SOCIEDADE BRASILEIRA DE CIRURGIA BARIÁTRICA E METABÓLICA (SBCBM), 2017).

A GV, que também é conhecida como cirurgia de Sleeve ou gastrectomia em manga, é um procedimento considerado restritivo e metabólico. Nessa técnica, o estômago assume o formato de um tubo, com capacidade reduzida ema até 100 mililitros $(\mathrm{mL})$. Apesar de não atuar de forma disabsortiva, a GV tem resultados considerados equivalentes ao BGYR em relação a mortalidade, perda de peso e resolução de comorbidades, mostrando-se uma excelente abordagem com indicação e sucessibilidade efetivas (CHUNG AY, et al., 2018; PETERLI R, et al., 2017; SBCBM, 2019). 
Apesar de apresentar uma baixa mortalidade, estima-se que 10 a $40 \%$ dos pacientes submetidos a cirurgia bariátrica apresentam complicações. Estes eventos adversos, que podem ser cirúrgicos, metabólicos e nutricionais, dividem-se em precoces e tardios, sendo os primeiros ocorridos nos primeiros 30 dias de pósoperatório e, o segundo, após este período. Entre as complicações precoces, citam-se as deiscências anastomóticas, falência renal, falência respiratória e óbito (FURLAN A, et al., 2019; MARCOTTE E e CHAND B, 2016).

Entre os eventos tardios mais recorrentes, destaca-se fístula na linha de grampeamento, sangramento gastrointestinal, obstrução intestinal, estenose de anastomose, ulceração marginal e fístula gastro-gástrica. Por fim, destaca-se a síndrome de dumping, a Doença do Refluxo Gastroesofágico (DRGE), a colelitíase, o diabetes mellitus e a osteopenia dentre as complicações metabólicas e nutricionais mais comuns (PALERMO $\mathrm{M}$, et al., 2015; ASSOCIAÇÃO BRASILEIRA PARA O ESTUDO DA OBESIDADE E DA SÍNDROME METABÓLICA (ABESO), 2016; FURLAN A, et al., 2019).

Logo, esta revisão de literatura teve como objetivo descrever e discutir as complicações pós-operatórias tardias mais comuns em cirurgia bariátrica, a partir da análise de duas técnicas amplamente utilizadas atualmente que são a Gastrectomia Vertical e a Cirurgia de Bypass Gástrico em Y de Roux.

\section{REVISÃo BIBLIOGRÁFICA}

A obesidade é um dos maiores desafios para os sistemas de saúde de diversos países, sobretudo para a saúde pública. Além disso, é uma patologia que está associada ao desenvolvimento de outras comorbidades como diabetes, hipertensão e dislipidemias, condições que representam cerca de $8 \%$ do total de gastos em saúde no Brasil (OMS, 2020; ABESO, 2016).

Segundo a OMS, em 2016, mais de 1.9 bilhões de adultos estavam acima do peso no mundo, sendo que 650 milhões eram obesos. No Brasil, a obesidade em adultos teve um aumento de $14.6 \% \mathrm{em}$ um decorrer de 17 anos, segundo a Pesquisa Nacional de Saúde (PNS) realizada pelo Instituto Brasileiro De Geografia e Estatística (IBGE) em 2019. De acordo com o estudo, foram contabilizados cerca de $25.9 \%$ de obesos acima de 18 anos e $6.7 \%$ em adolescentes de 15 a 17 anos, havendo prevalência no sexo feminino em ambos os casos (IBGE, 2020). Dados de outra importante pesquisa nacional, a Vigilância De Fatores de Risco e Proteção para Doenças Crônicas por Inquérito Telefônico (VIGITEL), constatou que, desde o início do monitoramento em 2006 até o ano de 2019, a prevalência de obesidade saltou de $11,8 \%$ para $20,3 \%$ no país (BRASIL, 2020).

Atualmente, o tratamento proposto para a obesidade envolve readaptação alimentar, o uso de fármacos, a psicoterapia e a prática de atividades físicas. Entretanto, quando o paciente não responde a esses tratamentos, a cirurgia pode ser indicada. Nesse contexto, a cirurgia bariátrica é um importante recurso nos casos de obesidade grave com falha documentada de tratamento clínico, proporcionando aos pacientes uma redução nos índices de mortalidade e melhora de comorbidades associadas (ZEVE JM, et al., 2012; ABESO, 2016).

Dessa forma, as indicações formais para operações bariátricas consistem em idade superior a 18 e inferior a 65 anos, IMC maior a $40 \mathrm{~kg} / \mathrm{m}^{2}$ ou $35 \mathrm{~kg} / \mathrm{m}^{2} \mathrm{com}$ uma ou mais comorbidades graves associadas (onde a perda de peso é capaz de melhorar a condição) e comprovação de que os pacientes não conseguiram perder peso ou manter a perda de peso apesar de cuidados médicos apropriados realizados há pelo menos dois anos (ABESO, 2016).

Existem vários procedimentos cirúrgicos diferentes e cada intervenção tem seu próprio perfil de vantagens e desvantagens. No geral, as cirurgias bariátricas podem ser classificadas em cirurgias restritivas e mistas. Nas cirurgias restritivas, somente o estômago é modificado de forma a provocar a redução do espaço para o alimento dentro da cavidade gástrica, gerando saciedade precoce. Nessa modalidade, as técnicas mais comumente realizadas são a gastroplastia vertical com bandagem, a bandagem gástrica ajustável por vídeo e o balão intragástrico e (SBCBM, 2017). 
Existem, ainda, as cirurgias mistas, onde estômago e o intestino são modificados. Neste tipo de procedimento existe o fator restritivo e um fator dissabsortivo pela diminuição do local de absorção de nutrientes no intestino delgado. Entre as técnicas mistas mais conhecidas estão a derivação biliopancreática com gastrectomia distal (Cirurgia de Scopinaro) e derivação gastrojejunal em Y-de-Roux, também conhecida como Cirurgia de Fobi-Capella (SBCBM, 2017; ZEVE JM, et al.2012).

Até o momento, não há um consenso claro sobre qual procedimento deve ser aplicado em cada caso. Atualmente, o Bypass Gástrico em Y de Roux (BGYR) é considerado o tratamento padrão ouro, mas esse procedimento é desafiado por outros procedimentos, como gastrectomia vertical (GV), sempre esperando encontrar procedimentos ainda mais eficientes, mas igualmente ou até mais seguros. Em relação a essas duas técnicas, estudos têm demonstrado que são igualmente eficientes em relação à perda de peso, metabolização de carboidratos, melhora das comorbidades e taxas de complicações (SCHLOTTMANN F e BUXHOEVEDEN R, 2018; BENAIGES D, et al., 2015; PETERLI R, et al., 2017; PETERLI R, et al., 2018).

A técnica de BGYR pertence ao grupo dos procedimentos mistos e é a mais realizada no Brasil. Nessa cirurgia é realizada a exérese do estômago proximal, limitando desse modo o seu volume a cerca de 10 a 25 $\mathrm{mL}$. Assim, o estômago remanescente, o duodeno e a parte inicial do jejuno ficam excluídos do trânsito alimentar, e o pequeno reservatório gástrico é então anastomosado a uma alça jejunal isolada em formato de Y, gerando, também, a má-absorção. O paciente submetido a essa técnica perde, em média, de $70 \%$ a $80 \%$ do excesso de peso inicial (ZEVE JM, et al., 2012; ABESO, 2016; SBCBM, 2017).

Já a GV, ou Sleeve, é uma técnica restritiva que era utilizada, inicialmente, apenas para casos de obesidade grave. Essa técnica é realizada por meio de uma incisão vertical do corpo gástrico, contiguamente à curvatura menor. Exclui-se a curvatura estomacal, o fundo gástrico, o corpo e o antro proximal, que correspondem a 70 a $80 \%$ da área gástrica total, o que leva a um esvaziamento gástrico rápido e a um trânsito intestinal acelerado. Ressalta-se também que é nesta área que ocorre a produção do hormônio grelina, relacionado intrinsecamente à fome e ao apetite. Logo, há uma redução dos níveis deste hormônio, quando comparado aos níveis basais, o que leva a uma consequente redução do apetite (RASPANTE LP, et al., 2020; MESUREUR L e ARVANITAKIS M, 2017; BENAIGES D, et al., 2015).

As complicações pós-operatórias mais comuns de todas as técnicas descritas são deiscência da sutura, estenoses, fístulas, infecções e hemorragia (mais comuns no pós-operatório precoce), hérnia interna e obstrução intestinal (mais comuns no pós-operatório tardio), além de tromboembolismo pulmonar. Nas técnicas disabsortivas e mistas há maior risco de ocorrência de diarreia com flatulência excessiva, desnutrição, deficiência de micronutrientes e doenças osteometabólicas (ABESO, 2016). Se as complicações não forem diagnosticadas e tratadas a tempo e corretamente, podem ocorrer sérios resultados, até mesmo a morte (HAIFU W, et al., 2017).

A incidência de fístula anastomótica é de 1-6\% após o BGYR e 3-7\% após a GV. Na cirurgia de BGYR, a ocorrência de fístula gastro-gástrica é considerada rara e geralmente ocorre devido à presença de úlcera gástrica entre a bolsa gástrica e o estômago excluído. A oclusão da fístula pode ser realizada com o tratamento clínico da úlcera e, em casos mais severos, pode ser necessário o fechamento cirúrgico por grampeamento desse pertuito que pode ser tanto por via videolaparoscópica quanto pela via laparotômica (ILIAS EJ, et al., 2015; PALERMO M, et al., 2015).

$\mathrm{Na}$ GV, a fístula na linha de grampeamento é uma das complicações cirúrgicas mais recorrentes e em $90 \%$ dos casos está localizada no nível superior do grampeamento (cárdia). Sua descoberta e intervenção é mais difícil do que na técnica do BGYR. Diferentes reforços na linha de grampeamento já foram testados, no entanto foram incapazes de conter a ocorrência de fístulas (BENAIGES D, et al., 2015; KASSIR R, et al., 2016).

A estenose anastomótica está relacionada principalmente à técnica de operação do cirurgião. No BGYR, $12 \%$ dos pacientes em média apresentam essa complicação que pode ocorrer em dois locais: na jejunojejunostomia e na gastrojejunostomia, sendo a gastrojejunostomia o lugar mais comum. Já na GV, geralmente ocorre no ângulo do estômago. Normalmente manifesta-se no prazo de 90 dias após a cirurgia, 
acompanhada de sintomas como vômitos pós-prandiais, com ou sem dor. A causa da formação da estenose pode incluir isquemia causando cicatrizes, formação de cicatriz excessiva não isquêmica, ulceração anastomótica recorrente, tensão ou mau posicionamento da anastomose. Os métodos de tratamento incluem dilatação endoscópica, colocação de stent e tratamento cirúrgico, se necessário (PALERMO M, et al., 2015; HAIFU W, et al., 2017; KASSIR R, et al., 2016).

Já a úlcera anastomótica após BGYR é uma úlcera péptica que ocorre na mucosa do jejuno, próximo à anastomose gastrojejunal e ocorre em 1-16\% dos pacientes. Úlceras também podem ocorrer no estômago remanescente após GV, principalmente na margem de ressecção do antro gástrico. A origem desta complicação é multifatorial incluindo tamanho da bolsa e sua orientação, isquemia da mucosa, linha de grampeamento rompida, fístula gastro-gástrica que permite o refluxo retrógrado de ácido através da fístula, reação a algum corpo estranho e substâncias exógenas (HAIFU W, et al., 2017; PALERMO M, et al., 2015).

A colelitíase também é um achado comum após a cirurgia bariátrica, ocorrendo entre $25 \%$ e $38 \%$ dos pacientes que perdem grande quantidade de peso. A manifestação desta patologia se dá por dor em hipocôndrio direito ou região epigástrica, cólicas de intensidade ascendente, dor de duração mais prolongada que os episódios prévios de cólica biliar, essa dor pode ser referida no ombro direito e irradiar-se para o dorso, pode ser relacionada a ingestão de alimentos, associado a febre, anorexia, vômitos e náuseas e em idosos, alteração do estado mental, icterícia ocorre em $20 \%$ dos casos (SANTOS JM, 2018).

O tratamento clínico desta complicação deve incluir dieta zero, hidratação venosa com reposição hidroeletrolítica, analgesia e antibioticoterapia. Havendo persistência de fator obstrutivo ou cálculo residual na via biliar, manter o antibiótico até que esta complicação tenha sido resolvida. Após isso, deve-se proceder a colecistectomia precoce por ser o tratamento definitivo para a colecistite aguda (SANTOS JM, 2018).

Outra complicação mais comumente encontrada após a cirurgia por BGYR é a ocorrência de hérnia e obstrução intestinal. A hérnia interna ou Hérnia de Petersen é uma complicação tardia do BGYR e ocorre em até $5 \%$ dos casos. No bypass, ao levar a alça intestinal para a realização da anastomose, há uma mudança na anatomia normal do paciente e esta alça passa a cruzar o cólon transverso. O contato do meso do intestino grosso com o meso da alça intestinal gerado na cirurgia provoca o surgimento de um espaço, chamado de brecha (espaço de Petersen). Há o aumento dessa alça, quando passa uma grande quantidade por esse espaço, ocorrendo uma distensão ou aumento das alças, causando uma hérnia interna que pode causar sintomas ou até complicações (KASSIR R, et al., 2016; PERIM CA, et al., 2019).

Dessa forma, a chegada de sangue e nutrientes ao intestino fica prejudicada, e se não tratadas a tempo, podem gerar necrose do intestino e perfurações, que podem se agravar. A hérnia interna pós-DGYR normalmente se apresenta como abdome agudo secundário a necrose ou perfuração intestinal, exigindo imediata exploração (por laparoscopia ou aberta) ou como forma de sintomas intermitentes como dor abdominal e vômitos. Nesses casos, o tipo de intervenção é cirúrgico, tentado, por laparoscopia, a simples redução da hérnia e sutura do defeito do mesentério é suficiente. Em raros casos, a ressecção do intestino se faz necessária (MEYER A e JEAN-LOUIS D, 2013).

Outra complicação comum após a cirurgia bariátrica, a síndrome de dumping, é vista particularmente após o BGYR, mas também pode ocorrer após a GV. É descrita em cerca de $75 \%$ dos pacientes e é caracterizada por dor abdominal, náuseas, problemas vasomotores (afrontamentos, sudorese, palpitações e diarreia), recusa alimentar e sonolência. Ela se desenvolve 30-60 minutos após uma refeição, mas pode ocorrer de forma tardia (90-240 minutos), e está associada a ingestão de alimentos doces ou hiperosmolares (SCARPELLINI E, et al., 2020).

A ingestão de alimentos ricos em açúcar ou carboidratos pode ocasionar o rápido esvaziamento gástrico de glicose, fazendo com que o fluido do componente intravascular seja deslocado para o lúmen intestinal gerando hipovolemia, aumento de contratilidade intestinal e atividade simpática compensatória, que tem papel central nos sintomas de dumping iniciais (dor, taquicardia, hipotensão). O trânsito rápido de glicose no intestino delgado causa, ainda, liberação maciça das incretinas polipeptídeo insulinotrópico glicosedependente (GIP) e glucagon-like peptide-1 (GLP-1) que estimulam a secreção exagerada de insulina 
causando hipoglicemia reativa, levando aos sintomas de dumping tardio (SCARPELLINI E, et al., 2020; KASSIR R, et al., 2016).

A doença do refluxo gastroesofágico, por sua vez, é considerada uma contraindicação para a realização da GV. Estudos apontam que a prevalência da DRGE aumentou após a realização desta técnica. Essa prevalência possui algumas causas potenciais, tais como hipotensão do esfíncter esofágico inferior, ruptura do ângulo de His, ressecção das fibras da tipoia, complacência gástrica reduzida com pressão intragástrica mais alta, diminuição do esvaziamento gástrico e ocorrência da hérnia de hiato (CHUNG AY, et al., 2018). Estudo realizado por Petreli R, et al. (2018) observou que a DRGE preexistente foi considerada significativamente melhor tratada pelo BGYR em comparação com a GV, demonstrando, assim, a importância da investigação sobre a existência dessa condição anteriormente à realização da cirurgia (MESUREUR $L e$ ARVANITAKIS M, 2017).

Por fim, sabe-se que deficiências nutricionais são comuns após a cirurgia bariátrica, sendo mais observadas nas cirurgias mistas que, além da restrição de volume ingerido, também interferem na absorção de nutrientes. Devido à redução gástrica, ocorre diminuição da digestão mecânica e da secreção de ácidos, o que, consequentemente, acarreta na má absorção de ferro e da vitamina B12. A exclusão da parte inicial do intestino delgado, por sua vez, causa má absorção de minerais, vitaminas lipossolúveis e hidrossolúveis, cálcio, magnésio, ferro e fator intrínseco. No BGYR, se a alça "Y" for longa, pode ocorrer deficiência de vitamina $D$ e, nesse caso, o risco a longo prazo é de osteoporose e osteomalacia (KASSIR R, et al., 2016; MOHAPATRA S, et al., 2020).

A deficiência de folato, de vitamina B12 e tiamina pode ocorrer em $26-66 \%$ dos casos e, por esta razão, a maioria dos pacientes submetidos a técnicas disabsortivas recebem complexos multivitamínicos, ferro, cálcio, vitamina $D$ e vitamina $B 12$ por toda a vida após cirurgia. O risco de desnutrição proteica também é maior no BGYR e também pode ocorrer em decorrência de vômitos prolongados ou má alimentação pós procedimento (KASSIR R, et al., 2016; LUPOLI R, et al., 2017).

\section{CONSIDERAÇÕES FINAIS}

De acordo com a literatura pesquisada, as técnicas Bypass Gástrico em Y de Roux e Gastrectomia Vertical são as mais utilizadas em cirurgias bariátricas hoje em dia, entretanto cada uma apresenta particularidades para indicação, bem como complicações em comum e específicas de cada técnica. Dentre as complicações pós-operatórias, as mais citadas são fístula, estenose, úlcera, hérnia, além das deficiências nutricionais. Logo, no momento de recomendação da cirurgia, é necessário avaliar os riscos do método cirúrgico a ser usado, o grau de obesidade do paciente, e, quando indicada a GV, deve-se certificar que o paciente não possui a doença do refluxo gastroesofágico. Atualmente, com o avanço da medicina, as técnicas cirúrgicas vêm sendo aprimoradas, objetivando reduzir cada vez mais o potencial risco que elas oferecem.

\section{REFERÊNCIAS}

1. ASSOCIAÇÃO BRASILEIRA PARA O ESTUDO DA OBESIDADE E DA SÍNDROME METABÓLICA (ABESO). Diretrizes brasileiras de obesidade. 4를. ed. São Paulo: ABESO, 2016.

2. BENAIGES D, et al. Laparoscopic sleeve gastrectomy: More than a restrictive bariatric surgery procedure? World journal of gastroenterology, 2015; 21(41): 11804-11814.

3. BRASIL. Vigitel Brasil 2019: Vigilância de fatores de risco e proteção para doenças crônicas por inquérito telefônico: estimativas sobre frequência e distribuição sociodemográfica de fatores de risco e proteção para doenças crônicas nas capitais dos 26 estados brasileiros e no Distrito Federal em 2019 . Disponível em: http://bvsms.saude.gov.br/bvs/publicacoes/vigitel_brasil_2019_vigilancia_fatores_risco.pdf. Acessado em: 9 abril 2021.

4. CHUNG AY, et al. Sleeve Gastrectomy: Surgical Tips. Journal of laparoendoscopic \& advanced surgical techniques, 2018; 28(8): 930-937.

5. FURLAN A, et al. Severe malnutrition after bariatric surgery and clinic manifestations of infection. Associação Médica Brasileira, 2019; 65(9): 1151-1155.

6. HAIFU W, et al. Prevention, diagnosis and treatment of perioperative complications of bariatric and metabolic surgery. Chinese Journal of Gastrointestinal Surgery, 2017; 20(4): 393-397. 
7. INSTITUTO BRASILEIRO DE GEOGRAFIA E ESTATÍSTICA (IBGE). Pesquisa nacional de saúde 2019: percepção do estado de saúde, estilos de vida, doenças crônicas e saúde bucal. 2020. Disponível em: https://biblioteca.ibge.gov.br/index.php/biblioteca-catalogo?view=detalhes\&id=2101764. Acessado em: 7 de abril de 2021.

8. ILIAS EJ, et al. Fístula gastro-gástrica pós-cirurgia de fobi-capella para tratamento de obesidade mórbida. Revista da associação médica brasileira, 2015; 56: 10.

9. KASSIR R, et al. Complications of bariatric surgery: Presentation and emergency management. International Journal of Surgery, 2016; $27:$ 77-81.

10. KOVESDY CP, et al. Obesidade e doenças renais: consequências ocultas da epidemia. Revista Brasileira de Pesquisas Médicas e Biológicas, 2017; 50(5): e6075.

11. LUPOLI R, et al. Bariatric surgery and long-term nutritional issues. World journal of diabetes, $2017 ; 8(11): 464-474$.

12. MARCOTTE E, CHAND B. Management and Prevention of Surgical and Nutritional Complications After Bariatric Surgery. Surgical Clinics of North America, 2016; 96(4): 843-856.

13. MESUREUR L, ARVANITAKIS M. Metabolic and nutritional complications of bariatric surgery: a review. Acta gastroenterologica Belgica, 2017; 80(4): 515-525.

14. MEYER A, JEAN-LOUIS D. Correção de hérnia laparoscópica: tela sem fixação é viável? Arquivos Brasileiros de Cirurgia Digestiva, 2013; 26(1): 27-30.

15. MOHAPATRA S, et al. Malnutrition in obesity before and after bariatric surgery. Disease-a-Month, 2020; 66(2): 100866.

16. ORGANIZAÇÃO MUNDIAL DA SAÚDE (OMS). Obesidade e Sobrepeso. $2020 . \quad$ Disponível em:https://www.who.int/en/news-room/fact-sheets/detail/obesity-and-overweight. Acessado em 5 de abril de 2021.

17. PALERMO M, et al. Complicações cirúrgicas tardias após Bypass gástrico: Revisão da literatura. Arquivos brasileiros de cirurgia digestiva, 2015; 28(2): 139-143.

18. PERIM CA, et al. Método simples para diagnosticar Hérnia de Petersen comprometendo a alça biliopancreática. Arquivos Brasileiros de Cirurgia Digestiva, 2019; 32(1): e1429.

19. PETERLI R, et al. Laparoscopic Sleeve Gastrectomy Versus Roux-Y-Gastric Bypass for Morbid Obesity-3-Year Outcomes of the Prospective Randomized Swiss Multicenter Bypass or Sleeve Study (SM-BOSS), Annals of surgery, 2017; 265(3): 466-473.

20. PETERLI R, et al. Effect of Laparoscopic Sleeve Gastrectomy vs Laparoscopic Roux-en-Y Gastric Bypass on Weight Loss in Patients With Morbid Obesity - The SM-BOSS Randomized Clinical Trial. JAMA. 2018; 319(3): 255-265.

21. RASPANTE LP, et al. Review and pictorial essay on complications of bariatric surgery. Revista Associação Médica Brasileira, 2020; 66(9): 1289-1295.

22. SANTOS JM. Colecistite aguda. Monografia (Residência Médica em Cirurgia Geral) - Hospital Geral de Fortaleza, Fortaleza, 2018; 17p.

23. SOCIEDADE BRASILEIRA DE CIRURGIA BARIÁTRICA E METABÓLICA (SBCBM). Tratamentos. 2017. Disponível em: https://www.sbcbm.org.br/tratamentos/. Acessado em: 18 de abril de 2021.

24. SOCIEDADE BRASILEIRA DE CIRURGIA BARIÁTRICA E METABÓLICA (SBCBM). Cirurgia Metabólica: Técnicas Cirúrgicas. 2019. Disponível em: https://www.sbcbm.org.br/tecnicas-cirurgicas-metabolica/ . Acessado em: 16 de abril de 2021.

25. SCARPELLINI E, et al. International consensus on the diagnosis and management of dumping syndrome. Nature reviews. Endocrinology, 2020; 16(8): 448-466.

26. SCHLOTTMANN F, BUXHOEVEDEN R. Laparoscopic Roux-en-Y Gastric Bypass: Surgical Technique and Tips for Success. Journal of laparoendoscopic \& advanced surgical techniques, 2018; 28(8): 938-943.

27. VILALLONGA R, et al. Operative and Postoperative Complications of Laparoscopic Sleeve Gastrectomy in Super and Nonsuper Obese Patients: A Center of Excellence Experience Comparative Study. Journal of Laparoendoscopic \& Advanced Surgical Techniques, 2020; 30(5): 501-507.

28. ZEVE JM, et al. Técnicas em cirurgia bariátrica: uma revisão da literatura. Revista Ciência \& Saúde, 2012; 5(2): 132140.

29. ZHANG Q, et al. Comparative risk of fracture for bariatric procedures in patients with obesity: A systematic review and Bayesian network meta-analysis. International journal of surgery, 2020; 75: 13-23. 